\title{
iPhysioMeter: A new approach for measuring heart rate and normalized pulse volume using only a smartphone
}

\author{
Kenta Matsumura • Takehiro Yamakoshi
}

Published online: 14 February 2013

(C) Psychonomic Society, Inc. 2013

\begin{abstract}
Heart rate (HR) and normalized pulse volume (NPV) are physiological indices that have been used in a diversity of psychological studies. However, measuring these indices often requires laborious processes. We therefore developed a new smartphone program, named iPhysioMeter, that makes it possible to measure beat-bybeat HR and ln NPV using only a smartphone. We examined its accuracy against conventional laboratory measures. Mental stress tasks were used to alter HR and ln NPV in 12 participants. Bland-Altman analyses revealed negligible proportional bias for HR and ln NPV or for their change values, expressed as $\triangle \mathrm{HR}$ and $\Delta \ln \mathrm{NPV}$. However, a relatively large fixed bias did emerge for ln NPV, as well as a small one for $\triangle \ln N P V$, although both were within the limits of agreement. These findings suggest that iPhysioMeter can yield valid measures of the absolute level of HR and of relative changes in ln NPV.
\end{abstract}

Keywords Electrocardiograph $\cdot$ Finger photoplethysmograph $\cdot$ iPhone $\cdot$ Mobile health

Heart rate (HR) and pulse volume (PV) are frequently used physiological measures in a wide range of psychological studies. Examples include studies of mental stress, health, mental disorders, emotion, and memory (e.g., Hamer, Tanaka, Okamura, Tsuda, \& Steptoe, 2007; Matsumura, Yamakoshi, Noguchi, Rolfe, \& Matsuoka, 2012; McNally et al., 2004; Sawada, Tanaka, \& Yamakoshi, 2001).

\footnotetext{
K. Matsumura $(\varangle) \cdot T$. Yamakoshi

School of Mechanical Engineering, College of Science and Engineering, Kanazawa University, Kakuma-machi, Kanazawa,

Ishikawa 920-1192, Japan

e-mail: kenta16moon@se.kanazawa-u.ac.jp
}

However, despite this widespread use, resource requirements and technical hurdles need to be overcome before these indices can be reliably and conveniently measured. These obstacles include the setting up of laboratory or ambulatory sensors and instruments, the application of analysis software, and the provision of the associated technical expertise. These requirements are often outside the core expertise of the user groups, who wish to focus their skills and effort on the application sphere.

Of direct relevance to this situation has been the rapid evolution of compact yet powerful computing and communication devices, notably the emergence of the smartphone. In addition to allowing for convenient mobile communication, the smartphone's processing power offers the possibility of measuring physiological indices using only the commercially available device itself (Jonathan \& Leahy, 2010, 2011; Scully et al., 2012). By using the lightemitting diode (LED) of the inbuilt flash as a light source and the complementary metal oxide semiconductor (CMOS) camera as a light sensor, the smartphone can be configured as a photo-plethysmograph (PPG) and, among other things, can then be used as a pulse wave recorder. Thus, HR and normalized PV (NPV), reported to be valid cardiovascular indices of autonomic nerve activity (Sawada et al., 2001), can be measured without using additional devices.

However, despite such progress, further significant developments are necessary. First, although the feasibility of calculating heart rate from the smartphone PPG has been shown, its experimental validation has not yet been carried out. Second, to the best of our knowledge, no reported studies have concerned the validation and use of the PV signal. Third, a beat-by-beat auto analysis program, to obviate the need for laborious manual analysis of the raw data, has not yet been made available for use with a smartphone. In this study, we therefore developed a smartphone program, named iPhysioMeter, equipped with all of the above- 
mentioned functions, and we then conducted an experiment in which HR and NPV were changed by means of psychological tasks. Our purpose was to examine the agreement of HR and NPV measurements derived from the iPhysioMeter and from conventional laboratory equipment.

\section{Methods}

\section{Participants}

A total of 12 university students (five women, seven men; age range $=21-24$ years), recruited via flyers, participated in this study. The main aim of the study was not hypothesis testing, but rather to determine the extent of agreement between two measurement techniques; we therefore used the same sample size as had been used in the first published NPV study (Sawada et al., 2001), under the condition that the number was even. The criteria for inclusion in the study were to be over 18 years of age, to have no history of or current cardiovascular disease, and to not be taking any prescription medication. No participant declared either a current or past history of being a smoker. Participants were asked in advance to refrain from any medication for $24 \mathrm{~h}$ before laboratory testing and to avoid the consumption of food and caffeine-containing substances, as well as intense physical activity, for $2 \mathrm{~h}$ before laboratory testing. The participants received about US $\$ 2.5$ for their participation. Written informed consent was obtained from participants after we had provided them with a complete description of the study. The ethics committee of Kanazawa University approved this study.

\section{Measurements and devices}

Laboratory measurements The beat-by-beat $\mathrm{HR}$ was derived from the $\mathrm{R}-\mathrm{R}$ interval ( $\mathrm{R}$ wave to $\mathrm{R}$ wave interval) of the electrocardiogram (ECG). Lead II of the ECG was obtained with disposal electrodes connected to standard bioamplifiers (John et al., 2009) built at the authors' laboratory.

NPV, an index of $\alpha$-adrenalin-mediated sympathetic activity (Sawada et al., 2001), was calculated by dividing the alternating-current (AC), or pulsatile, component of the finger PPG by the direct-current (DC) component. The finger PPG was measured in the transmittance mode using an 810-nm near-infrared LED as the light source and a photodiode as the photo sensor, placed on opposite sides of the tip of the left middle finger. The sensors and an amplifier to which the sensors were connected were built in the authors' laboratory, as has been previously reported (Tanaka et al., 2011). A logarithmic transformation was applied to the NPV values (i.e., ln NPV) to normalize the distribution.
All signals were sampled at a rate of $1 \mathrm{kHz}$ with a resolution of 16 bits using an analog-to-digital converter (National Instruments, USB-6218) and stored digitally in a computer (Dell, Latitude 5430).

iPhysioMeter iPhysioMeter is a program that was developed using the Xcode 4.3.3 developer tool (Apple, Inc., Cupertino, $\mathrm{CA}$ ), and designed to run on iPhone $4,4 \mathrm{~S}$, or 5 smartphones running with iOS 5.1 (Apple, Inc.) or later. ${ }^{1}$ The measurement principle is based on the reflectance mode PPG, where the camera flash LED serves as the light source and the CMOS image sensor (camera) serves as the photo sensor. In the present study, the sampling rate was approximately $30 \mathrm{~Hz}$ (30 fps), which is the maximum speed allowed for iPhones 4 and $4 \mathrm{~S}$, and the resolution of the light intensity detected by the CMOS camera was 8 bits each for red, green, and blue light at each of the $192 \times 144$ pixels (Apple, Inc., 2011). Although the pulse wave could be measured using any of the three light colors (red, green, or blue), we chose to use the green light, because previous studies have consistently shown its superiority over red and blue in terms of the relative freedom from motion artifacts and high signal/noise ratio (Jonathan \& Leahy, 2011; Maeda, Sekine, \& Tamura, 2011). Green light detected by the CMOS camera was averaged among all of the $192 \times 144$ pixels, and then artifacts were minimized by lowpass filtering implemented in software to produce the PPG DC component. The AC component was separated from the DC component using a software high-pass filter.

The application is run by tapping its icon on the iPhone's home screen. Then the flash LED and CMOS camera must be gently covered with the tip of the left index finger (Fig. 1a), whereupon measurement will start automatically after a 6-s delay period, during which time the CMOS camera is calibrated. This calibration is accomplished by using the camera calibration functions of iOS with the setting of white balance mode fixed. Once the measurement process is established, the detected pulse waves are displayed on the screen, and realtime beat-by-beat analysis is carried out (Fig. 1b). HR is calculated from the peak-to-peak intervals in the pulse wave. NPV is subsequently derived by dividing the foot-to-peak amplitude of the $\mathrm{AC}$ component by the $\mathrm{DC}$ component; the latter is not seen on the display. Values departing significantly from the most recent 10 -s data trend are treated as outliers. The 10 -s interval was determined on the basis of the $0.1-\mathrm{Hz}$ characteristic frequency of Mayer waves (Julien, 2006). All beat-by-beat data are stored on the iPhone's memory. To stop or restart measurement, it is only necessary to touch the stop/start button displayed on the screen, or to press the stop/start hardware button of the wired headphone, normally

\footnotetext{
${ }^{1}$ The iPhysioMeter software is available for free at the iTunes App store, where this program is distributed worldwide.
} 
Fig. 1 (a) Initial screen of iPhysioMeter; how to position the index finder on the iPhone is depicted, with a brief description. (b) Typical example of the main screen of iPhysioMeter. The pulse wave measured is displayed in real time across the screen. The numerical results of the online beat-by-beat analysis of heart rate (HR) and normalized pulse volume (NPV) are displayed in the center and are also shown in the lower part. bpm, beats per minute; a.u., arbitrary units a

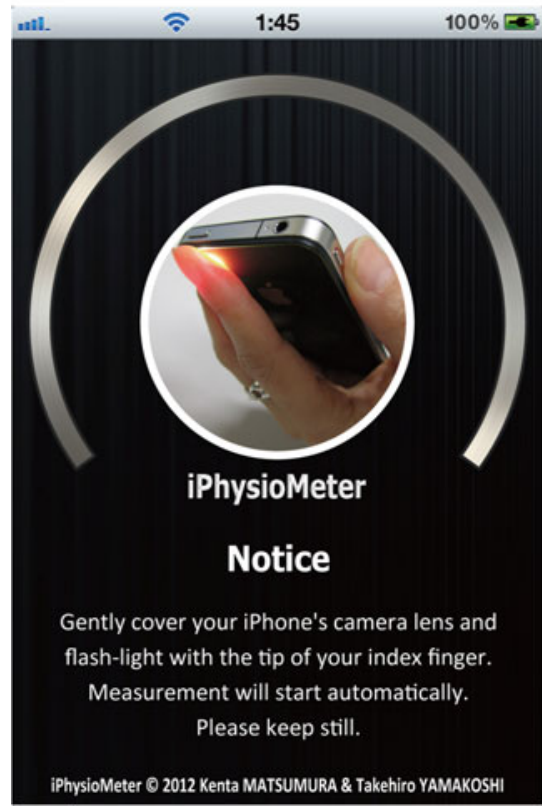

b

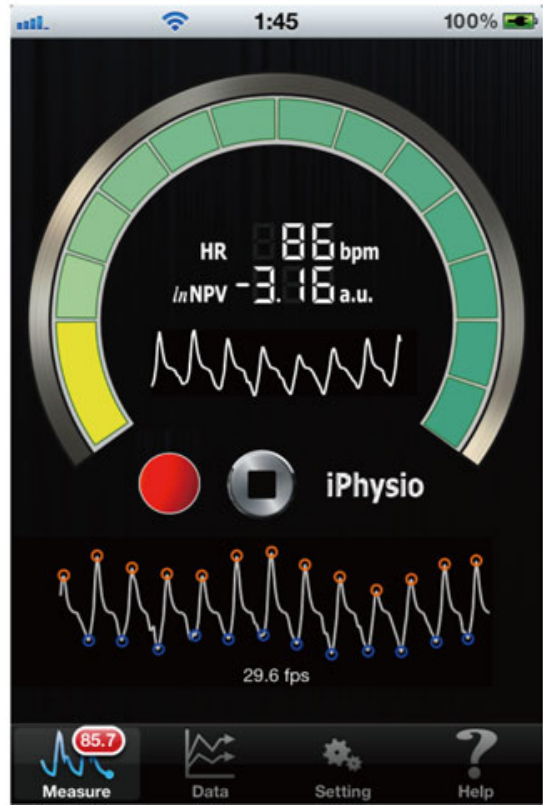

provided with an iPhone or available as a third-party product. Once finished measuring, the stored data can be sent by e-mail via Wi-Fi or a Bluetooth wireless network.

\section{Procedure}

The experiment was performed in a $2 \times 5 \mathrm{~m}$, soundattenuated experimental room, maintained at a temperature of $27-28^{\circ} \mathrm{C}$. Although this temperature seems rather high, all of the participants felt comfortable wearing light summer clothing. First, participants sat in a reclining chair in front of an LCD monitor (EIZO, FlexScan S1721), keeping their left arm at heart level and the palm of the hand upward. The LCD monitor and a headphone (Sony, MDR-CD900ST) were used to present stimuli. Next, the sensors and instruments were attached to the participant, who was then asked to hold an iPhone 4S (Apple, Inc.) on which iPhysioMeter was installed. The participant was then asked to keep as still as possible in order to minimize movement artifacts. Only one iPhone $4 \mathrm{~S}$ was used in this study, and when it was measuring, no other applications were running in the background. The display of the iPhone was monitored by a further LCD monitor using an HDMI adapter. Finally, the left hand of the participant was covered with a black cloth to prevent him or her from looking at the iPhone's display.

The experimental procedure adopted in this study was essentially identical to that used in the first published NPV study (Sawada et al., 2001). The experiment began with a 10min rest period, the last 3 min of which were used as a baseline (BL). In this period, the participant was asked to sit still and to observe the blank LCD screen in which the stimulus would be shown. Next, the participant performed two kinds of mental stress task, one a 3-min period of mental arithmetic (MA), and the other a 3-min period of mirror tracing (MT). These two tasks were selected to evoke somewhat different physiological responses. In the MA task, the participants were required to subtract 13 sequentially from 5,000 (thus, 4,987, 4,974, 4,961, . . .) as quickly and accurately as possible (see, e.g., Matsumura et al., 2012). In the MT task, they were required to trace a narrow, star-shaped track presented on the LCD clockwise as quickly and accurately as possible, without deviation, using a cross-hair cursor on the LCD, which moved as the mirror image of a track pad (Magic Trackpad, Apple, Inc.; see, e.g., Sawada, Nagano, \& Tanaka, 2002). These tasks were separated by a 5-min rest interval, and their order was changed and equalized across the participants. iPhysioMeter was rebooted between conditions.

\section{Data analysis}

Beat-by-beat HR and In NPV values were averaged for each 1min period. Then, delta change $(\Delta)$ values were calculated by subtracting the first 1-min BL value from the remaining two BL, three MA, and three MT values so as to conduct detailed analyses.

All 1-min average data were further averaged to produce single BL, MA, and MT values. These values were compared using a series of one-way repeated measures analysis of variance (ANOVA) tests. The Greenhouse-Geisser correction was applied to the degrees of freedom, where appropriate. For post-hoc comparison, Tukey HSD tests were used to compare the differences among conditions.

To evaluate the correspondence between the two measurements, geometric mean regression (Ludbrook, 1997) and Bland-Altman analysis (Bland \& Altman, 1986) were used. 
Table 1 Physiological variables during three conditions measured by two methods

\begin{tabular}{|c|c|c|c|c|c|c|c|c|}
\hline \multirow[t]{3}{*}{ Measures } & \multicolumn{3}{|l|}{ Condition } & \multicolumn{5}{|c|}{ Results of Statistical Test } \\
\hline & \multirow{2}{*}{$\begin{array}{l}\mathrm{BL} \\
M(S D)\end{array}$} & \multirow{2}{*}{$\begin{array}{l}\text { MA } \\
M(S D)\end{array}$} & \multirow{2}{*}{$\begin{array}{l}\mathrm{MT} \\
M(S D)\end{array}$} & \multicolumn{4}{|c|}{ ANOVA } & \multirow[t]{2}{*}{ Tukey $H S D$} \\
\hline & & & & $F_{(2,22)}$ & $\varepsilon$ & $p$ & $\eta_{p}^{2}$ & \\
\hline \multicolumn{9}{|l|}{ Laboratory device } \\
\hline HR (bpm) & $71.0(9.6)$ & $86.7(14.7)$ & $75.1(12.3)$ & 36.07 & .56 & $<.001$ & .77 & $\mathrm{MA}>\mathrm{MT}, \mathrm{BL}$ \\
\hline ln NPV (a.u.) & $-3.48(0.47)$ & $-4.44(0.44)$ & $-4.22(0.47)$ & 89.84 & & $<.001$ & .89 & $\mathrm{MA}<\mathrm{MT}<\mathrm{BL}$ \\
\hline \multicolumn{9}{|l|}{ iPhysioMeter } \\
\hline HR (bpm) & $71.2(9.8)$ & $86.8(14.6)$ & $75.4(12.1)$ & 36.93 & .56 & $<.001$ & .77 & $\mathrm{MA}>\mathrm{MT}, \mathrm{BL}$ \\
\hline ln NPV (a.u.) & $-4.48(0.44)$ & $-5.26(0.48)$ & $-5.01(0.48)$ & 41.89 & & $<.001$ & .79 & $\mathrm{MA}<\mathrm{MT}<\mathrm{BL}$ \\
\hline
\end{tabular}

Note. $\mathrm{HR}=$ heart rate, $\mathrm{NPV}=$ normalized pulse volume, $\mathrm{BL}=$ baseline, $\mathrm{MA}=$ mental arithmetic, $\mathrm{MT}=$ mirror tracing.

In the former analysis, a slope, intercept, and correlation coefficient (Pearson's $r$ ) were calculated, whereas in the latter, the mean of the differences (fixed bias) and correlation coefficient between the differences and averages (proportional bias) were calculated. In each analysis, a total of 108 1-min data pairs (three conditions $\times$ three 1-min periods $\times 12$ participants) were used. Analyses were carried out using IBM SPSS Statistics 19.0 (IBM) and Microsoft Excel for Mac 2011.

\section{Results}

HR and ln NPV values during BL, MA, and MT

The mean values of HR and ln NPV during each period, together with the results of statistical analysis, are summarized in Table 1.

\section{Agreement of the two measuring methods}

Scatterplots displaying paired measures of $\mathrm{HR}, \Delta \mathrm{HR}, \ln$ $\mathrm{NPV}$, and $\Delta \ln \mathrm{NPV}$, along with their Bland-Altman plots, are shown together in Fig. 2. The outcomes of geometric mean regressions and Bland-Altman analyses are summarized in Table 2.

\section{Discussion}

In this study, we examined the agreement of physiological indices (HR and NPV) derived simultaneously from the newly developed iPhysioMeter smartphone program and from laboratory equipment, during the conduct of two mental stress tasks. The agreement of the two measurement methods for $\mathrm{HR}$ and $\Delta \mathrm{HR}$ was found to be quite high. Only negligible fixed and proportional biases emerged, and the ranges of the limits of agreement in Bland-Altman plots were small. In addition, the HR responses to mental stress tasks were comparable between the two methods. Therefore, these findings suggest that iPhysioMeter yields valid measures of HR and $\triangle \mathrm{HR}$

In contrast, the agreements for $\ln$ NPV and $\triangle \ln$ NPV were not as consistent as those for HR and $\Delta \mathrm{HR}$. Although only negligible proportional biases were apparent, we found a relatively large fixed bias for $\ln \mathrm{NPV}$, although it was within the limits of agreement. First, this systematic error most likely arose from the differences in the measuring configurations, such as wavelength (near-infrared vs. green light) and measuring mode (transmittance vs. reflectance). It is known that these configurations can have a substantial influence on $\ln$ NPV via differences in the absorption coefficients of hemoglobin and melanin pigment and those in the pathway of light from light source to sensor (Giltvedt, Sira, \& Helme, 1984; Sawada et al., 2001). Second, it is possible that this systematic error could have arisen from the adverse effects of finger-iPhone contact pressure during measurement. A certain degree of contact pressure is a prerequisite for smartphone measurements, including iPhysioMeter, but this pressure is practically negligible for laboratory measurements, where the sensors are attached directly to the finger. It has been reported that arterial pressure can have a substantial effect on In NPV (Tanaka et al., 2011), and it also seems plausible that finger-iPhone contact pressure could affect arterial pressure at the measuring site. Therefore, we

Fig. 2 (Left) Scatterplots of each index measured by the iPhysioMeter against those measured by the corresponding conventional laboratory devices among all of the 108 data pairs (three conditions $\times$ three 1-min periods $\times 12$ participants). (Right) Bland-Altman plots of each index, measured by iPhysioMeter and by conventional laboratory devices. $\mathrm{HR}$, heart rate; NPV, normalized pulse volume; $\Delta$, delta change values; GMR, geometric mean regression; ECG, electrocardiograph; PPG, photo-plethysmograph; bpm, beats per minute; a.u., arbitrary units 
$H R$
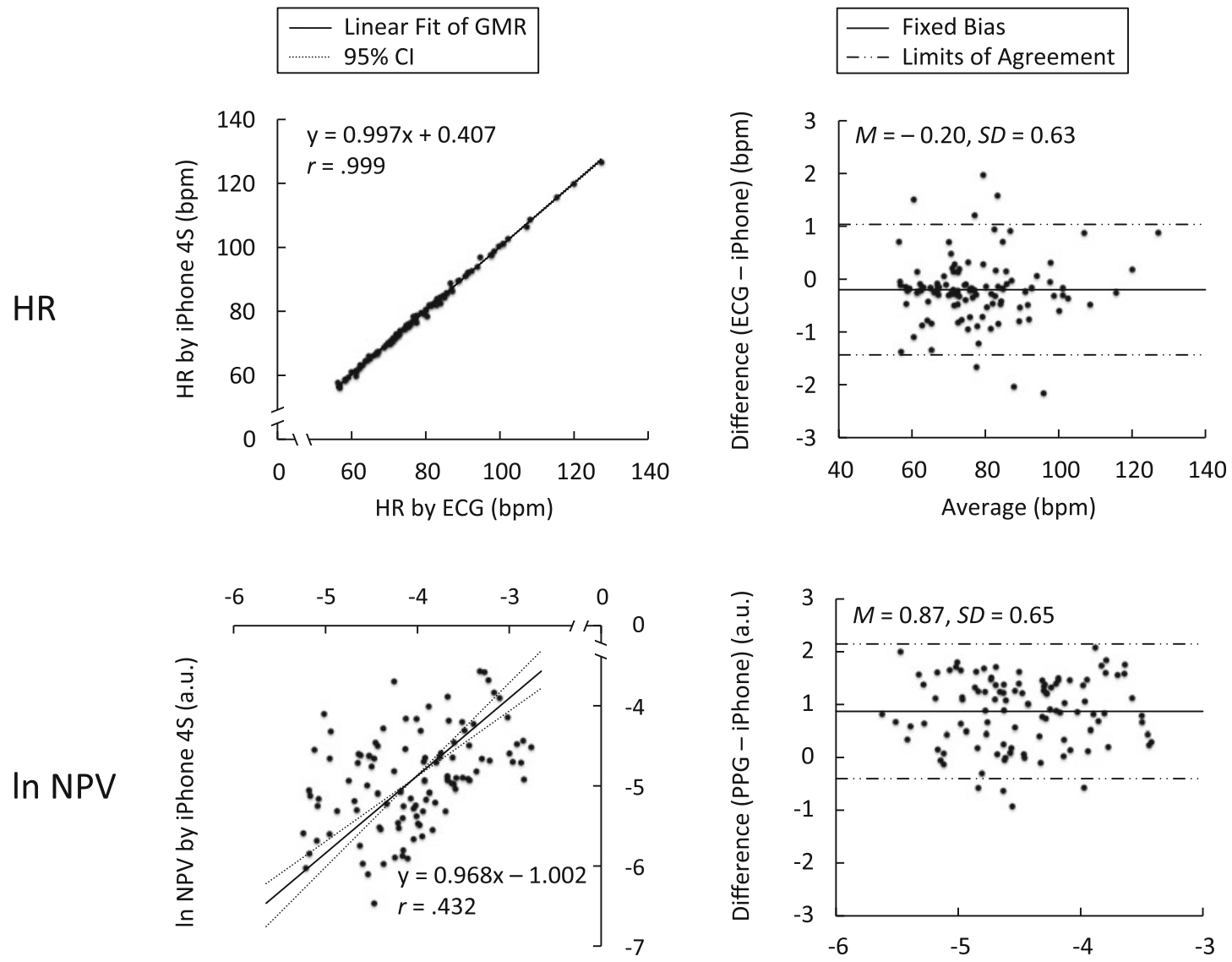

In NPV by PPG (a.u.)
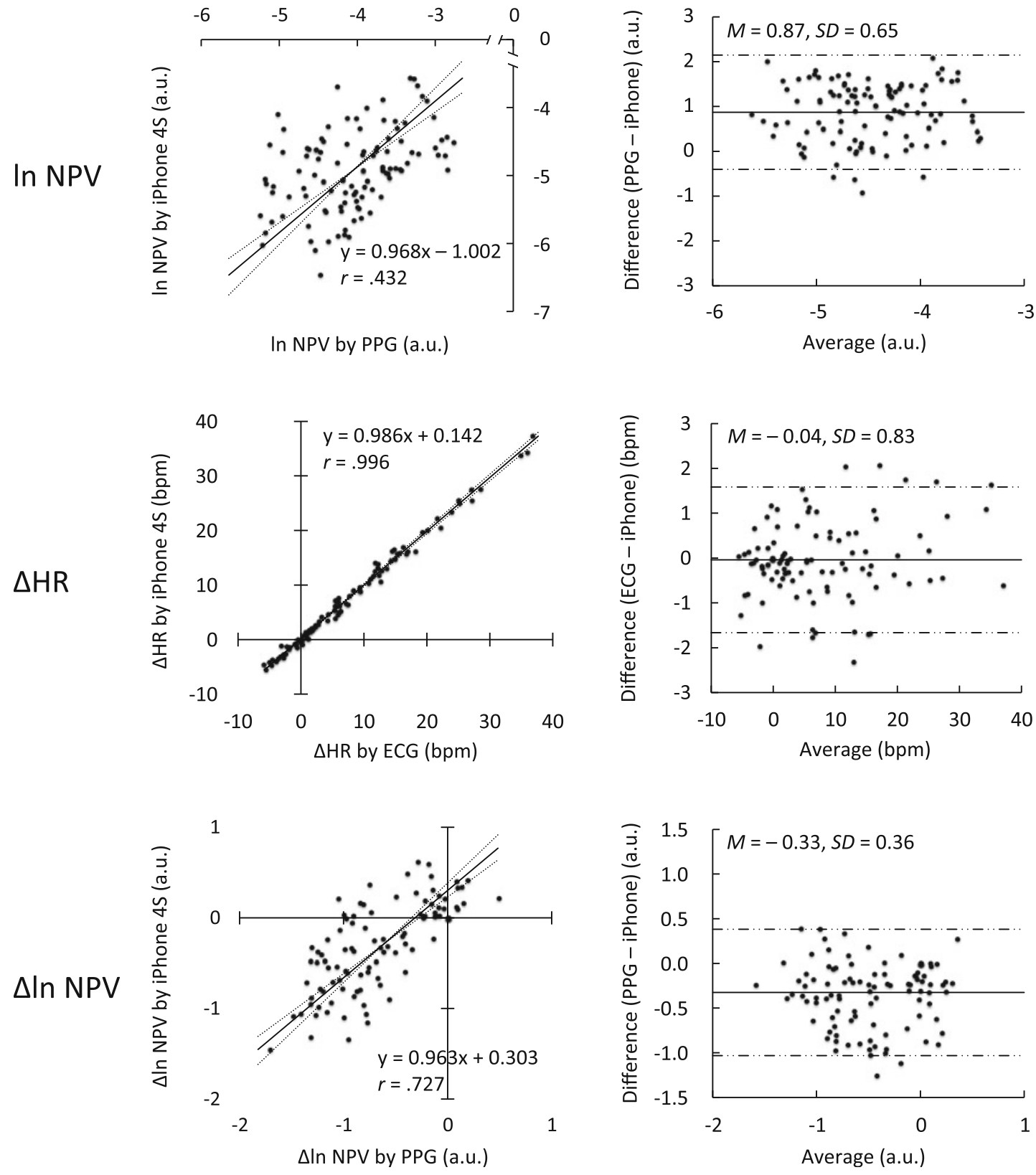
Table 2 Outcomes of geometric mean regression analyses and Bland-Altman plots

\begin{tabular}{|c|c|c|c|c|c|c|}
\hline \multirow[t]{2}{*}{ Measures } & \multicolumn{3}{|c|}{ Geometric Mean Regression } & \multicolumn{3}{|c|}{ Bland-Altman Plot } \\
\hline & $\begin{array}{l}\text { Slope } \\
{[95 \% \mathrm{CI}]}\end{array}$ & $\begin{array}{l}\text { Intercept } \\
{[95 \% \mathrm{CI}]}\end{array}$ & $\begin{array}{l}r \\
{[95 \% \mathrm{CI}]}\end{array}$ & $\begin{array}{l}M \\
{[95 \% \mathrm{LOA}]}\end{array}$ & $S D$ & $\begin{array}{l}r \\
{[95 \% \mathrm{CI}]}\end{array}$ \\
\hline HR & $\begin{array}{l}0.997 \\
{[0.989,1.006]}\end{array}$ & $\begin{array}{l}0.407 \\
{[-0.261,1.069]}\end{array}$ & $\begin{array}{l}.999 \\
{[.9985, .9993]}\end{array}$ & $\begin{array}{l}-0.20 \\
{[-1.43,1.03]}\end{array}$ & 0.63 & $\begin{array}{l}.060 \\
{[-.131, .246]}\end{array}$ \\
\hline $\ln \mathrm{NPV}$ & $\begin{array}{l}0.968 \\
{[0.814,1.150]}\end{array}$ & $\begin{array}{l}-1.002 \\
{[-1.623,-0.264]}\end{array}$ & $\begin{array}{l}.432 \\
{[.265, .574]}\end{array}$ & $\begin{array}{l}0.87 \\
{[-0.40,2.14]}\end{array}$ & 0.65 & $\begin{array}{l}.037 \\
{[-.153, .224]}\end{array}$ \\
\hline$\Delta \mathrm{HR}$ & $\begin{array}{l}0.986 \\
{[0.969,1.002]}\end{array}$ & $\begin{array}{l}0.142 \\
{[0.027,0256]}\end{array}$ & $\begin{array}{l}.996 \\
{[.995, .997]}\end{array}$ & $\begin{array}{l}-0.04 \\
{[-1.67,1.58]}\end{array}$ & 0.83 & $\begin{array}{l}.167 \\
{[-.023, .345]}\end{array}$ \\
\hline$\Delta \ln \mathrm{NPV}$ & $\begin{array}{l}0.963 \\
{[0.844,1.099]}\end{array}$ & $\begin{array}{l}0.303 \\
{[0.230,0.385]}\end{array}$ & $\begin{array}{l}.727 \\
{[.624, .805]}\end{array}$ & $\begin{array}{l}-0.33 \\
{[-1.03,0.38]}\end{array}$ & 0.36 & $\begin{array}{l}.055 \\
{[-.135, .241]}\end{array}$ \\
\hline
\end{tabular}

Note $. \mathrm{HR}=$ heart rate, $\mathrm{NPV}=$ normalized pulse volume, $\Delta=$ delta change values, $\mathrm{LOA}=$ limits of agreement.

might reasonably conclude that finger-iPhone contact pressure, via subsequent arterial pressure change, can exert an influence on $\ln$ NPV that is by no means negligible. When we take these considerations together, it seems that the origin of the fixed bias is an inherent feature of smartphone measurements, and thus is inevitable for iPhysioMeter.

Besides the fixed biases, larger variations emerged in $\ln$ NPV and $\Delta \ln$ NPV than in HR and $\Delta$ HR. Although, in general, such random error could arise from one or more unknown sources, in this particular case, finger-iPhone contact pressure probably again exerted a harmful effect on the accuracy of the measurement. That is, the contact pressure served as a random error, in that it would not be constant across participants. It is likely that some users would press the iPhone firmly, whilst others would do so lightly, but it is difficult to control this variation. Interestingly, random error was still evident in $\triangle \ln$ NPV even after canceling out this kind of variation by conducting the delta change calculation. This was likely due to the fact that the participants could not maintain constant contact pressure throughout the measurement process. Meanwhile, HR was free from such a problem because this chronometric index can be calculated regardless of PV amplitude, so long as the finger-iPhone pressure does not occlude the arteries completely.

The existence of fixed bias and random error does not necessarily negate the usefulness of $\ln$ NPV being measured by iPhysioMeter. This is because physiological indices may often exhibit absolute level inaccuracies and/or large individual differences that are irrelevant to psychological factors. Thus, psychologists examining condition differences or Condition $\times$ Group interactions using physiological indices prefer to adopt a within-subjects or mixed designs and/or to use a change in value - namely, reactivity - to avoid this problem in advance (e.g., Hamer et al., 2007; Matsumura et al., 2012; McNally et al., 2004). This supports the view that the existence of fixed biases will not be detrimental in many cases, and on balance, ln NPV measured by iPhysioMeter actually fulfills the necessary requirements. In fact, as is shown in Table 1, the ln NPV responses to mental stress tasks were comparable between the two methods, which was probably due to there being only negligible proportional biases in $\ln$ NPV and $\triangle \ln$ NPV. Moreover, even though the variation was larger in ln NPV than in HR, the sensitivity of ln NPV measured by the iPhysioMeter to these tasks $\left(\eta_{\mathrm{p}}{ }^{2}=.79\right)$ was comparable to that of HR measured by conventional laboratory devices $\left(\eta_{\mathrm{p}}{ }^{2}=.77\right)$. This is consistent with the opinion of Sawada et al. (2001) that PV is quite sensitive to a wide range of psychological stimuli. In normal situations, large sample sizes or the averaging of repeated measurements are necessary to reduce such random error, but NPV's high sensitivity to tasks obviates this necessity. Therefore, when an absolute level of ln NPV measurement is not necessary-which is true in the majority of cases-iPhysioMeter yields valid measures of $\ln$ NPV and $\triangle \ln$ NPV.

In addressing the limitations of the iPhysioMeter, two issues should be noted. First, the iPhysioMeter program calculates HR on the basis of pulses detected at the finger, and therefore, in the case of arrhythmias, it may not yield accurate results. For example, in the case of pulse deficit, the HR derived from the iPhysioMeter would be lower than that from the ECG. However, a careful examination of the beatby-beat data would help detect such problems. Second, our experiment was conducted on a limited population in a small number of situations, so further studies dealing with more diverse populations and situations will be needed. Despite these limitations, iPhysioMeter can provide researchers with a measurement environment for accurate absolute-level HR and relative-change-level ln NPV, and we hope that it will make a positive contribution to a wide range of psychological studies.

Author note The authors give special thanks to Ken-ichi Yamakoshi, Kanazawa University, for his helpful comments on this work, and to Peter Rolfe, Oxford BioHorizons Ltd and Harbin Institute of 
Technology, for his helpful comments and for his assistance in preparing the manuscript. We also give sincere thanks to Lee Jihyoung and Kenshi Yoshimoto, Kanazawa University, for their technical assistance in conducting the experiment. The study was partially supported by the Ministry of Education, Culture, Sports, Science and Technology, Japan via Grant-in-Aid for Young Scientists (A) No. 24680063. K.M. developed the program, conceived and designed the study, performed the experiment and the analysis, and drafted the manuscript. T.Y. developed the program, conceived and designed the study, performed the analysis, and helped to draft the manuscript. The authors declare that they have no competing interests.

\section{References}

Apple, Inc. (2011). AV foundation programming guide (iOS Developer Library, Oct 12, 2011 Ed.). Retrieved from http://developer. apple.com/library/ios/DOCUMENTATION/AudioVideo/ Conceptual/AVFoundationPG/Articles/00_Introduction.html

Bland, J. M., \& Altman, D. G. (1986). Statistical methods for assessing agreement between two methods of clinical measurement. Lancet, 1, 307-310. doi:10.1016/S0140-6736(86)90837-8

Giltvedt, J., Sira, A., \& Helme, P. (1984). Pulsed multifrequency photoplethysmograph. Medical and Biological Engineering and Computing, 22, 212-215. doi:10.1007/BF02442745

Hamer, M., Tanaka, G., Okamura, H., Tsuda, A., \& Steptoe, A. (2007). The effects of depressive symptoms on cardiovascular and catecholamine responses to the induction of depressive mood. Biological Psychology, 74, 20-25. doi:10.1016/j.biopsycho.2006.06.003

John, W. C., Jr., Michael, R. N., Walter, H. O., Robert, A. P., Frank, P. P., Jr., Melvin, P. S., et al. (2009). Medical instrumentation application and design (4th ed.). Hoboken, NJ: Wiley.

Jonathan, E., \& Leahy, M. (2010). Investigating a smartphone imaging unit for photoplethysmography. Physiological Measurement, 31, N79-83. doi:10.1088/0967-3334/31/11/N01

Jonathan, E., \& Leahy, M. J. (2011). Cellular phone-based photoplethysmographic imaging. Journal of Biophotonics, 4, 293-296. doi:10.1002/jbio.201000050
Julien, C. (2006). The enigma of Mayer waves: Facts and models. Cardiovascular Research, 70, 12-21. doi:10.1016/ j.cardiores.2005.11.008

Ludbrook, J. (1997). Comparing methods of measurements. Clinical and Experimental Pharmacology and Physiology, 24, 193-203. doi:10.1111/j.1440-1681.1997.tb01807.x

Maeda, Y., Sekine, M., \& Tamura, T. (2011). The advantages of wearable green reflected photoplethysmography. Journal of Medical Systems, 35, 829-834. doi:10.1007/s10916-0109506- $\mathrm{Z}$

Matsumura, K., Yamakoshi, T., Noguchi, H., Rolfe, P., \& Matsuoka, Y. (2012). Fish consumption and cardiovascular response during mental stress. BMC Research Notes, 5, 288. doi:10.1186/17560500-5-288

McNally, R. J., Lasko, N. B., Clancy, S. A., Macklin, M. L., Pitman, R. K., \& Orr, S. P. (2004). Psychophysiological responding during script-driven imagery in people reporting abduction by space aliens. Psychological Science, 15, 493-497. doi:10.1111/j.09567976.2004.00707.X

Sawada, Y., Nagano, Y., \& Tanaka, G. (2002). Mirror tracing and the provocation of vascular-dominant reaction pattern through heightened attention. Journal of Psychophysiology, 16, 201-210. doi:10.1027/0269-8803.16.4.201

Sawada, Y., Tanaka, G., \& Yamakoshi, K. (2001). Normalized pulse volume (NPV) derived photo-plethysmographically as a more valid measure of the finger vascular tone. International Journal of Psychophysiology, 41, 1-10. doi:10.1016/S01678760(00)00162-8

Scully, C. G., Lee, J., Meyer, J., Gorbach, A. M., Granquist-Fraser, D., Mendelson, Y., et al. (2012). Physiological parameter monitoring from optical recordings with a mobile phone. IEEE Transactions on Bio-Medical Engineering, 59, 303-306. doi:10.1109/ TBME.2011.2163157

Tanaka, G., Yamakoshi, K., Sawada, Y., Matsumura, K., Maeda, K., Kato, Y., et al. (2011). A novel photoplethysmography technique to derive normalized arterial stiffness as a blood pressure independent measure in the finger vascular bed. Physiological Measurement, 32, 1869-1883. doi:10.1088/ 0967-3334/32/11/003 